\title{
IMPROVING IMAGE RESOLUTION THROUGH THE CRA ALGORITHM INVOLVED RECYCLING PROCESS TO IMAGE RECONSTRUCTION
}

\author{
S. N. Tazi ${ }^{1}$, Deepika Verma ${ }^{2}$, Vinesh Jain ${ }^{3}$, A. K. Dubey ${ }^{4}$, Rakesh Rathi ${ }^{5}$ \\ ${ }^{1,2}$ M.Tech (Student) CSE \& IT \\ ${ }^{3,4,5}$ Assistant Professor (CSE Dept) \\ ${ }^{1,4}$ Member IEEE \\ Govt. Engineering College Ajmer-305001, Rajasthan (INDIA)

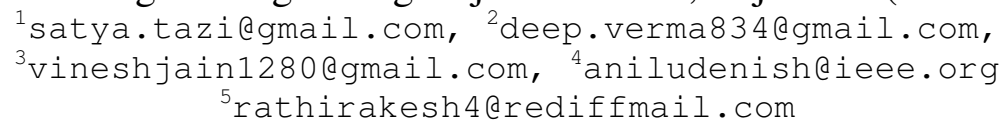

\begin{abstract}
Image processing concepts are widely used in medical fields. Digital images are prone to a variety of types of noise. Noise is the result of errors in the image acquisition process for reconstruction that result in pixel values that reflect the true intensities of the real scenes. A lot of researchers are working on the field analysis and processing of multi-dimensional images. Work previously hasn't sufficient to stop them, so they continue performance work is due by the researcher. In this paper we contribute a novel research work for analysis and performance improvement about to image resolution. We proposed Concede Reconstruction Algorithm (CRA) Involved Recycling Process to reduce the remained problem in improvement part of an image processing. The CRA algorithms have better response from researcher to use them.
\end{abstract}

\section{KEYWORDS}

image, noise, acquisition, reconstruction, CRA, visualization.

\section{INTRODUCTION}

Visual perspective of object is the current theme for computers device to make them more effective for vision. There is the potential to sense body position, head orientation, direction of gaze, pointing commands, and gestures. Interactive vision is continuous growth to the computer application. A game player didn't think to press the button every time (or they didn't want to directly connect the computer physical want to directly connect the computer physically), they required automated vision system through that sensor automatically sense out the activities (action) movement done by game player is recognised. A CAD designer may be tiered due to the labour work for physically connect to computer for manipulate object through their finger and hands. Similarly different machine application user is also suffering too many types of action listener. The Concept of computer vision and human computer interaction make the machine David C. Wyld (Eds) : ICCSEA, SPPR, CSIA, WimoA - 2013 
interaction safety, joyful and interesting. They reduce lot of risk and few challenges occurred to achieve their motive. Their application response time is touch the user vision thought. The algorithm for computer vision is designed to create the base line for bulk people reliability of their vision and also reduce the cost factor of existing system.

Now a day's researcher and academic both are emerging to highlight the previous work of image processing and search novel inventory for recon sting the user action. To catch the exact action of user is more difficult and their reorganisation, reconstruction is so complex. There for lot of researcher are involving to tackle to this goal but can't achieve so continues research is going now. The vision input function as part of larger system that include the interactive graphics display. They mainly focus to control different application such as computer game, television program graphically they control and show to the user that objects exist in the real world. Here we focus on various fundamental of visual measurement. In incremental order of complexity large object tracking reorganisation of shape edges and motion analysis of object to track them. We are proposed to different approaches for tracking the object and maximise the reorganisation of object [1].

\subsection{Digital images Vision}

The representation of image object in two dimensional grid is in the form of row * column (size $\mathrm{m} * \mathrm{n}$, where $\mathrm{m}$ for row $\& \mathrm{n}$ for column). $\mathrm{m}^{*} \mathrm{n}$ shown the pixel of images which have a value to encode the colour and intensity of image. E.g. the RGB colour image pixel representation in triplet [R; G; B]. Respectively Red, Green \& Blue colour repenting the gray level image. In pixel form contain single value corresponding to their intensity [2].

- Input Image: The initial phase of image processing is image acquisition. The sensors of kinetic camera sense-out the image object in different projection and collect all intensity of image data.

- Pre-processing: The systematically arrangement of multiple captured image data is known as pre-processing stage. This phase also cover the manipulation (e.g. enhancement) and reduction of noise.

- Segmentation: In this phase we categorise the whole image in many parts such as edge and region of images. So that expected changes might be done accordingly needed.

- Reconstruction of data: the edge and region of image data is converted from series of $\mathrm{m}$ dimensional to $\mathrm{n}$-dimensional. Both dimension $\mathrm{M}$ and $\mathrm{N}$ are matched to their region with edge.

- Matching: the registration of all n-dimensional data are putted in an order so that, the combination made useful information. This method is applying on data from distinguishing sources or single source applied to different times.

- Recognition: The recognition of object does not achieved in a signal phase of identification of element that may depend on the final purpose of the analysis.

The pipelining of computer vision \& image analysis pipeline is as follows:- 


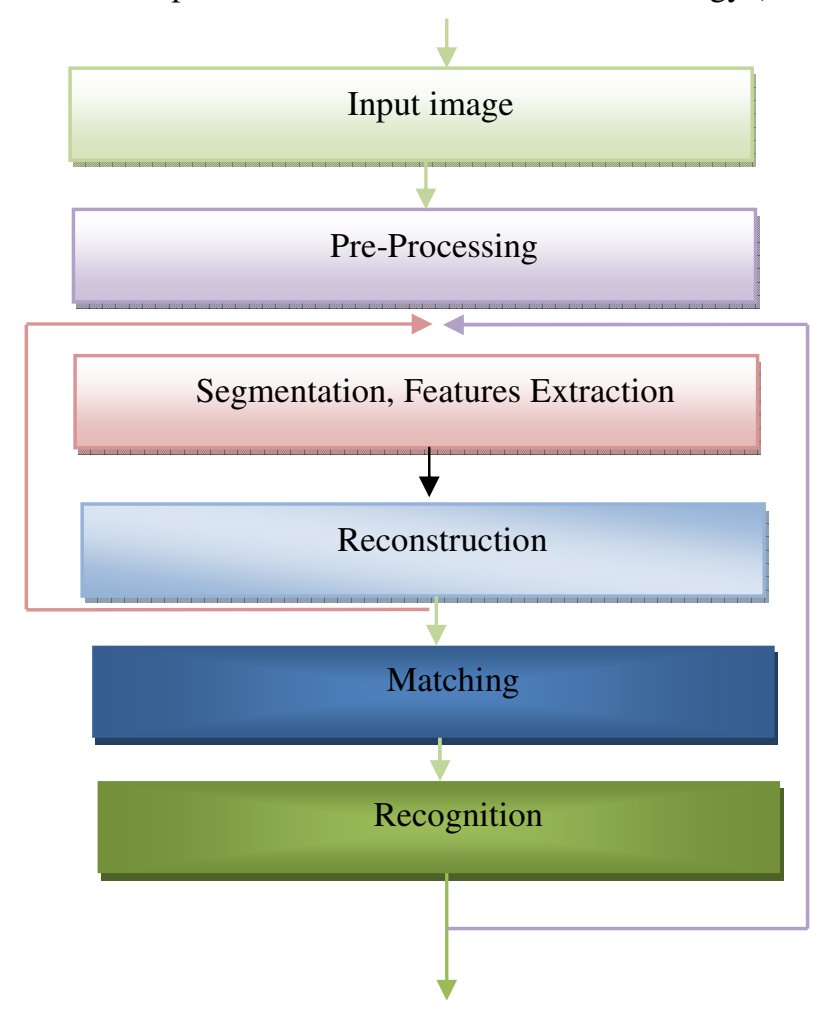

Fig.1: Generic image analysis and computer vision pipeline [3]

\section{Projective Capturing TeChNiQue}

\subsection{Calibration}

A most common projective capturing technique is camera calibration. It involves the estimation of both side camera parameter such that extrinsic and intrinsic. The calibration of multiple views set up two cameras for computing different parameter of object (basic calibration algorithm). Camera parameter estimation is a practice varies approach to calibrating the employed object. The correction of lens distortion required calibration rig (known as geometry), to estimate the camera based parameter object. i.e., check board pattern. The position and it's orientation of internal parameter camera is calculated through the different perspective view of check board using calibration algorithm. The strong calibration of camera object is a process to compute different view calibration of camera parameter which must be addresses as follows:-

1. Capture N (at least 3) images with the checkerboard pattern and estimate pointcorrespondences.

2. Estimate and correct the radial lens distortion.

3. For each captured image, calculate the $\mathbf{N}$ homographic transforms.

4. Using the $\mathrm{N}$ homographic transforms, calculate the intrinsic and extrinsic parameters.

5. Refine the calculated camera parameters as explained in Section D above. 
The correction method of non-linear radial lens distortion for calibration process given more accrued result to obtain homographic $\mathrm{H}_{\mathrm{i}}$ in compared to standard technique. This we consider $\left(h^{\mathrm{m}}\right)^{\mathrm{T}}=\mathrm{h}^{\mathrm{mT}}$, where first one represent the multiplication of inner product and another one is a equation to lead a scalar.

\subsection{Epipolar Geomatry}

To estimate the depth information simulate according to human eyes. We required stereo vision simulation technique. As we know that real world object is projected into $2 \mathrm{D}$ coordinate. Through camera real world projection technique describe as two point $\mathrm{X}$ and $\mathrm{Y}$ in the given figure the projection of point $X$ is pointed out the object surface. Space point $X$ of object is projected in 2D coordinate by using of two different cameras $\mathrm{C} 1$ and $\mathrm{C} 2$ which pointed out $\mathrm{x}$ and $x^{\prime}$ our projection point to respective camera. The line that connects $C 1$ and $C 2$ points is baseline. The point's $e$ and $e^{\prime}$ on the baseline are epipolar poles. Any point in real world space compose different epipolar plane with two projection plane. But the baseline and epipolar poles were never changed. The lines crossed by the epipolar and projection planes are epipolar lines $l 1$ and $l 2$.

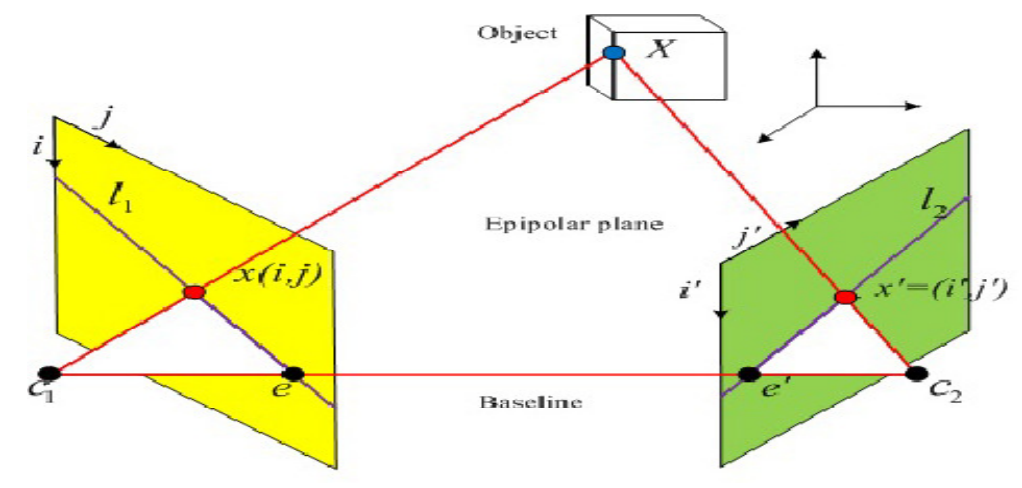

Fig. 2 Image Processing 1) Left image 2) Right image [5]

Any point on the epipolar plane will be projected on the epipolar lines $l 1$ and $l 2$. According to this rule we can reduce the researching area to find out the projection point the two images.

\section{FILTERING TECHNIQUE}

Noise: anything that make changes in the vision of original images also known as image noise. To remove noises from those images is the part of filtering technique. By the use of this classical and novel filtering technique we achieved the nearest copy of original image. Few of common filtering technique are preferred for image noise as follows:-

\section{(i) Low pass filtering}

The simplest and easiest method of filtering to decrease the image noise is known as low pass filtering .This method is applying to calculate the average image noise and minimal filter to removal them. For the work of minimal filtering it's called Gaussian or average filtering. 
(ii) High pass filtering

In the case of minimal filtering the sharpness of image can't recover its original form (At the blurred and complex image noise). To reduce such a problem we use the maximum filtering to reconstruct the originality of image noise. This is known as high pass filtering method.

The above both filtering method are the part of linear operation taken at Image noise. In the case of Non-linear operations other filtering method such as median or out-of-range filters are applied. A special class of algorithm for low pass filtering is applied to enhance the object desirable. Consider many degraded and translated and rotation of object is available for particular application. The idea behind these motive are called correlation averaging which align the elements in two given standard as correlation technique to estimate the internal relation between objects and another one is the calculate the average of that object correlation. The special class of resolution of images are based on signal-to-noise ratio. The resolution indirectly grows up on the basis of square root of the number of averaged individual element. On the basis of distortion of images relevant correction are required for example Geometric distortion call to relevant Geometric correction that is the necessity of image registration.

\section{RECONSTRUCTION}

\subsection{Basic Concept of Reconstruction}

Let us suppose that we want to share our experience and wonderful momentum to our friends and relatives, and then we capture the images in different angles and shared to them. The principle behind capturing different angle images is occurred in our mind cause of distortion of single views image. These thinking phenomena of different angle images captured by camera generate the basic concept of reconstruction.

A systematic projection of acquired images for reconstruction as users view shown in figure below:-
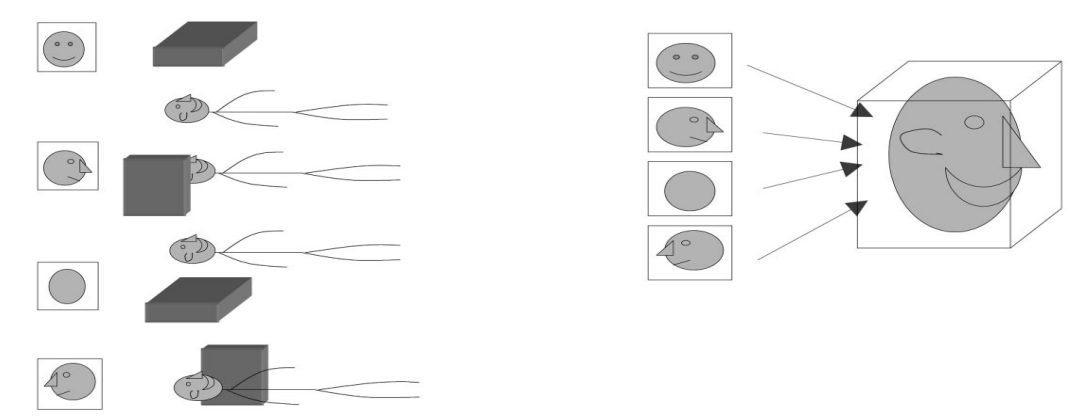

Fig.3 Projection data acquired from different views is used to reconstruct the image. [4]

\subsection{Definition of Reconstruction}

Geometric reconstruction is defined as construction of damaged images and multiple views of images fusion from rebuild to redesign. An acquired view of images categories the reconstruction in different terms.

These terms are follows: 
(1)Substitutions

(2) Typing with constraint sets (type equation)

(3)Unification solving constraint sets.

(4) Principle types

(5) Let Polymorphism

\subsection{Basic Algorithms for Reconstruction}

1) Filtered back-projection algorithm: "A standard mathematical procedure to distribute the equal amount of activities in every section of projection for every angle, to reconstruct the original image for spike back technique is known as back projection algorithm“. Through the help of back projection algorithm we get blurred version of original image.

Proposed Approach:-

Let us assume that

$$
\sigma \mathrm{B}(\mathrm{I})=\mathrm{C} \tilde{=} \mathbf{A} \text {. }
$$

$\mathrm{A}=$ original image.

$\mathrm{B}=$ Back projection Method

$\mathrm{C}=$ Reconstructed image.

$\mathrm{I}=$ Noise image.

$\sigma$ is standard constant depending on angle/degree rotation.

For a maximum reconstruction

6 is nearest to $180^{\circ}$.

2) Analytical algorithms: In this algorithm, the Fourier transform and its inverse are taken for the iteration. These algorithms use the basic concept of back projection algorithm.

3) Three-dimensional image reconstruction: The formation of three-dimensional (3D) image is constructed through the help of 2D images slices. The access plan measurement is composed by both back projection filtered and filtered back-projection algorithm.

4) Iterative reconstruction: The iterative reconstruction algorithm is most popular in nuclear medicine because of following reasons:-

(a) For the low counts the algorithm is simple to model and manage projection noise.

(b) Simply modelled the imaging physics (i.e., non-uniform attenuation, geometry, scatter etc.) 


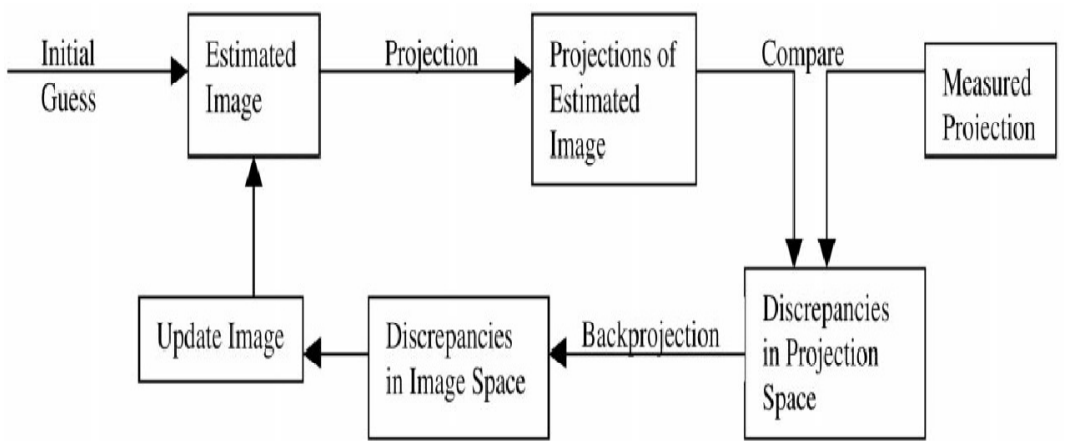

Fig.4. Flow chart of iterative image reconstruction scheme. [4]

5) Eight-point linear algorithm: For creating essential matrix in estimation of computer vision, eight point algorithms are used. Appropriate given matrix shown the stereo vision camera pair to the corresponding point of image.

\section{CONCEDE RECONSTRUCTION AlgORITHM}

\subsection{Proposed CRA}

Step1. Capture bundle of images from multiple cameras

Step2. Pre-Processing

Step3. Apply Noise Removing Technique

3.1 if noise $==$ Gaussian noise(salt-and-pepper or speckle)

Removing Noise By Linear Filtering

Else if noise $==$ blur

3.2 Removing Noise by Adaptive Filtering

Else

3.3 Removing Noise by Median Filtering

Step4. Apply any segmentation techniques

4.1 Clustering methods

4.2Compression-based methods

4.3 Histogram-based methods

4.4 Edge detection

4.5 Region-growing methods

4.6 Split-and-merge methods

4.7 Partial differential equation-based methods

4.8 parametric methods

4.9 Level set methods

4.10 Fast Marching methods

4.11Graph partitioning methods

4.12Watershed transformation and

4.13 Multi-scale segmentation 
Step5. Partition of images at aspect levels

\{

Complete Image/4=A,

$\mathrm{A} / 4=\mathrm{B}$,

$\mathrm{B} / 4=\mathrm{C}$,

$\mathrm{Nth} / 4=æ \quad$ (aspect level)

\}

Step6. Render æ images

Step7. Reconstruct render images

$$
\mathrm{Rc}=\sum_{0}^{\mathrm{Nth}} \mathfrak{x}
$$

Step8. Compare reconstructed image from original image

$\mathrm{Rc}=\mathrm{Ro}$

Step9. if noise is occurred

Then Backtracking step 3

$$
\mathrm{Rc} \neq \mathrm{Ro}
$$

Else step 10

\section{Step10.Exit}

In this algorithm we are capturing a bundle of image from multiple cameras and applying perprocessing technique to give the systematic arrangement and applying noise removal techniques for enhancement of image. For applying CRA algorithm we are classified region and edges of an image. After found of edges and region we are dividing whole image by four this process do continuously until user accepted level is achieved. The rendering process is applicable to those achieved object. The render object of image is walking through the reconstruction techniques. After reconstruction of an image compared have to original image. Recycling this process until comparison made equivalent. 


\subsection{Flow Chart of CRA}

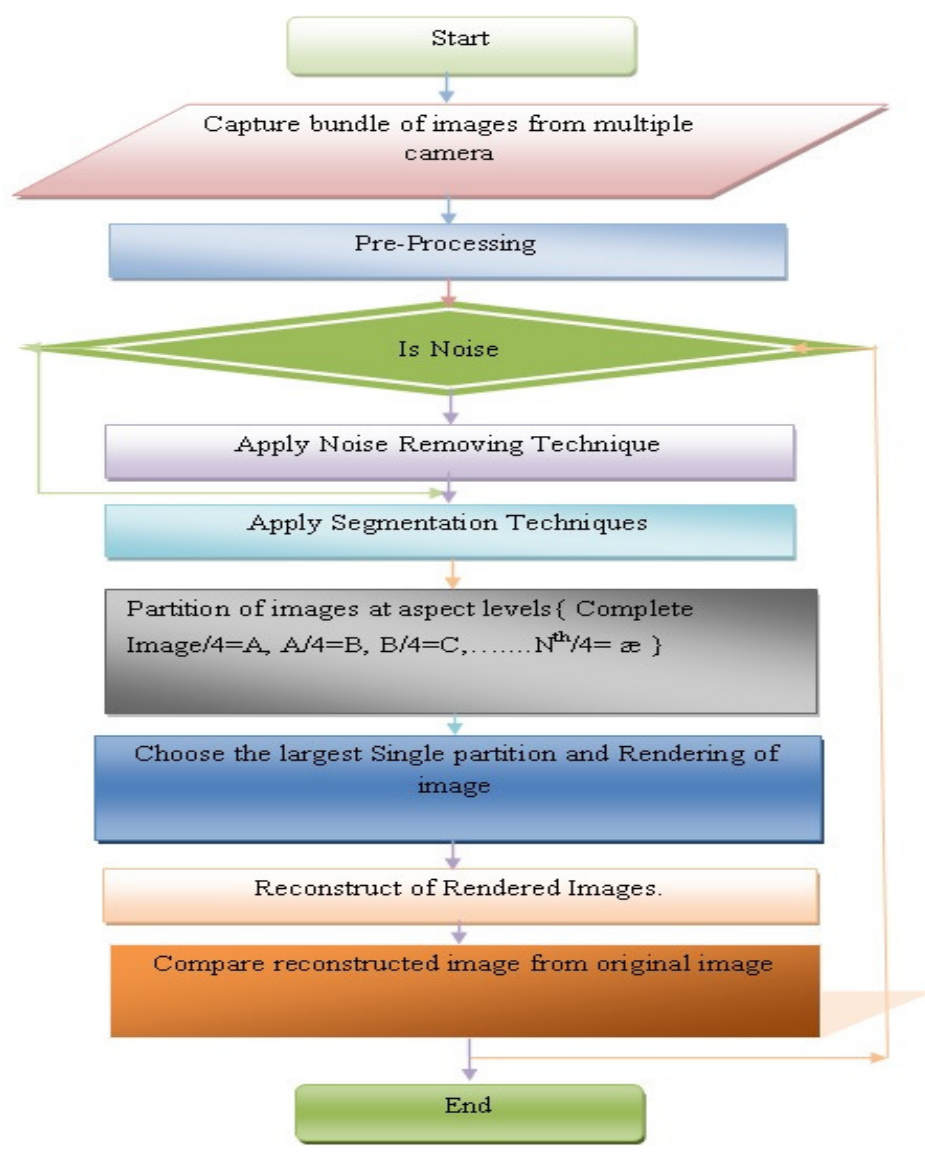

Fig. 5 Proposed Model of CRA

\section{CONCLUSION}

The graphics, animation and visualization are most important terms used in daily life. HCI involve the better human vision through improvement of visualization of picture /images. We study the different terms in image processing from historical perspective and find out there projective capturing technique i.e, calibration and epipolar geometry are commonly used. Two filtering techniques (low pass and high pass) are used to remove the noise from blurred image. For the improvement of image visualization many reconstruction algorithm are used. all of these technique based on back projection algorithm. The available algorithm does not provide image visualization at aspect level. To remove such a problem we proposed a novel algorithm Title as "Concede Reconstruction Algorithm (CRA)". The CRA algorithm improved the visualization of image object with aspect ratio. 


\section{ACKNOWLEDGMENTS}

The author wishes to thank faculty staffs for helpful comments and for help in preparing the manuscript. They thank his class-met for encouragement and support at GECA. Document is drawings \&prepared by Sh. Anil K Dubey, S.N. Tazi\& Deepika Verma.

\section{REFERENCES}

[1] M. Roth, K. Tanaka, C. Weissman, W. Yerazunis,. Computer Vision for Interactive Computer Graphics, TR99-02 January 1999, Mitsubishi Electric Research Laboratories.

[2] Daniel Vaqueroa, Matthew Turka, Kari Pullib, Marius Ticob, Natasha Gelfandb, A survey of image retargeting techniques, Nokia Research Center 955 Page Mill Road Palo Alto, CA, 94304-1003, USA, SPIE 7798, 7798142010.

[3] Thierry Pun, Guido Gerig and Osman Ratib, Image Analysis and Computer Vision in Medicine, into Computer vision in medicine, February 8, 1993.

[4] G.L. Zeng, Image reconstruction - a tutorial, Computerized Medical Imaging and Graphics 252001.

[5] Deepak Gaur, Yaduvir Singh, Raj Kumar Sagar, 3D Reconstruction of Uniform Curved Object Model From 2D Images, International Journal of advancement in electronics and computer engineering (IJAECE) Volume 1, Issue1, April 2012, pp.1-3, ISSN 2278 - 1412. 